Research Article

\title{
Existence, Uniqueness, and Exponential Stability of Uncertain Delayed Neural Networks with Inertial Term: Nonreduced Order Case
}

\author{
M. Iswarya $\mathbb{D}^{1},{ }^{1}$ R. Raja $\mathbb{D},{ }^{2}$ Q. Zhu $\mathbb{D},{ }^{3,4}$ M. Niezabitowski $\mathbb{D},{ }^{5}$ J. Alzabut $\mathbb{D},{ }^{6,7}$ \\ and C. Maharajan ${ }^{8}$ \\ ${ }^{1}$ Department of Mathematics, Alagappa University, Karaikudi 630 003, India \\ ${ }^{2}$ Ramanujan Centre for Higher Mathematics, Alagappa University, Karaikudi 630 003, India \\ ${ }^{3}$ School of Mathematics and Statistics, Hunan Normal University, Hunan 410 081, China \\ ${ }^{4}$ School of Information Science and Engineering, Chengdu University, Chengdu, 610106, China \\ ${ }^{5}$ Faculty of Automatic Control, Electronics and Computer Science, Department of Automatic Control, and Robotics, \\ Silesian University of Technology, Akademicka 16, Gliwice 44-100, Poland \\ ${ }^{6}$ Department of Mathematics and General Sciences, Prince Sultan University, Riyadh 11586, Saudi Arabia \\ ${ }^{7}$ Group of Mathematics, Faculty of Engineering, Ostim Technical University, 06374 Ankara, Turkey \\ ${ }^{8}$ Department of Mathematics, V.S.B Engineering College, Karur, India
}

Correspondence should be addressed to Q. Zhu; zqx22@126.com

Received 31 January 2021; Revised 15 March 2021; Accepted 29 March 2021; Published 7 May 2021

Academic Editor: Li Haitao

Copyright (c) $2021 \mathrm{M}$. Iswarya et al. This is an open access article distributed under the Creative Commons Attribution License, which permits unrestricted use, distribution, and reproduction in any medium, provided the original work is properly cited.

In this work, we mainly focus on uncertain delayed neural network system with inertial term. Here, the existence, uniqueness, and exponential stability of inertial neural networks are derived without shifting the second order differential system into first order through substituting variables. Initially, we construct a proper Lyapunov-Krasovskii functional to investigate the stability of novel uncertain delayed inertial neural networks, which is different from the classical Lyapunov functional approach. By utilizing the Kirchhoff's matrix tree theorem, Cauchy-Schwartz inequality, homeomorphism theorem, and some inequality techniques, the necessary and sufficient conditions are derived for the designed framework. Subsequently, to exhibit the strength of this outcome, we framed a quantitative example.

\section{Introduction}

In recent years, because of the fruitful applications in various domains such as gesture recognition [1], image quality enhancement [2], secure communication [3], face detection [4], image compression [5] and medical image processing [6], the dynamical behaviour of different kinds of neural networks (NNs), namely, Cohen-Grossberg neural networks (CGNNs) [7], recurrent neural networks (RNNs) [8, 9], Hopfield neural networks [10], bidirectional associative memory neural networks (BAMNNs) [11], and chaotic neural networks (CNNs) [12], have been studied widely. In addition to that, time delays of a dynamical framework play a supreme role in various disciplines. Because of the finite propagation velocity, time delays are unavoidable in signal transmission of neural networks, which may lead the unwanted dynamical responds such as chaotic and bifurcation. Marcus and Westervelt [13] firstly proposed the stability analog of neural networks with time delays in 1989.

$$
\dot{x}_{i}(t)=-a_{i} x_{i}(t)+\sum_{j=1}^{n} b_{i j} f_{j}\left(x_{j}(t)\right)+\sum_{j=1}^{n} c_{i j} f_{j}\left(x_{j}(t-\tau)\right)+I_{i},
$$

where $x_{i}(t) \in \mathbb{R}^{n}$ indicates the position of the ith neuron, $a_{i}>0$ is the positive constant weight, $\left(b_{i j}\right)_{n \times n},\left(c_{i j}\right)_{n \times n}$ indicates the transmission weight matrices from ith neuron to $j$ th neuron, and $f_{j}: \mathbb{R}^{n} \longrightarrow \mathbb{R}^{n}$ characterize the transfer function, the constant delay $\tau>0$, and $I_{i}$ denotes the external input. In the nervous system, the time-delays occur due to 
the propagation time of neurotransmitters forming presynaptic neurons to postsynaptic neurons. The time delays are occurring not only in constant manner [14] but also according to the number of parallel pathways with different lengths and sizes of the axon; it may be classified into various kinds such as discrete $[15,16]$, distributed, and mixed delays [17-19].

$$
\dot{x}_{i}(t)=-a_{i} x_{i}(t)+\sum_{j=1}^{n} b_{i j} f_{j}\left(x_{j}(t)\right)+\sum_{j=1}^{n} c_{i j} \int_{t-\sigma(t)}^{t} f_{j}\left(x_{j}(s)\right) \mathrm{d} s+I_{i},
$$

where the term $\sigma(t)$ signifies the distributed time-varying delay. Besides the aforementioned delay kinds, there is another kind of delay called proportional delay, which explains that, $\delta(t)=t-q_{i j} t$, where $q_{i j}>0$, for $i, j=1,2, \ldots, n$, pantograph delay factor with $0<q_{i j}<1$. This kind of delay is also know as discrete unbounded delay since the delay term $\delta(t) \longrightarrow+\infty$ as $t \longrightarrow+\infty$. Due to the constrains of the continuously distributed delay, the proportional delay is less conservative.

$$
\dot{x}(t)=-a_{i} x_{i}(t)+\sum_{j=1}^{n} b_{i j} f_{j}\left(x_{j}(t)\right)+\sum_{j=1}^{n} c_{i j} f_{j}\left(x_{j}\left(q_{i j} t\right)\right)+I_{i} .
$$

Moreover, this type of delay is used in web quality of service (QoS) routing decision [5]. Due to its applications in the areas such as wireless LAN [20], network application servers [21], and integro differential equations [22], the study on proportional delay increases the research interest of researchers; in [23], the synchronization of CGNNs with proportional delays was studied on the basis of Lyapunov theory, the exponential synchronization of INNs with proportional delays and reaction diffusion was investigated under Lyapunov--Krasovskii functional and Wirtinger inequality in [24]; in [25]. the exponential stability of CNNs with proportional delay was investigated by utilizing the matrix theory and Lyapunov theory. Furthermore, in recent days, the stability analysis of dynamical systems is a hot research topic [26, 27].
In recent years, most of the studies are mainly focused on first order derivative of the NNs, although it is great significant to introduce the inertial term in NNs because of the reason that the influence of the inductance, which was firstly investigated in electronic neural networks and found that the coupling of the neurons is in inertial way; the dynamics can be complex $[28,29]$. In 1997, Wheeler and Schieve initially proposed the inertial neural networks [30].

$$
\ddot{x}_{i}(t)=-a_{i} \dot{x}_{i}(t)-b_{i} x_{i}(t)+\sum_{j=1}^{n} c_{i j} f_{j}\left(x_{j}(t)\right)+I_{i} .
$$

Here, $\ddot{x}_{i}(t)$ denotes the inertial term and $b_{i}>0$ is a positive constant. In recent years, the convergence analysis of INNs has been focused by lot of research scientists, for instance by utilizing the Lyapunov functional method, inequality techniques, and analytical method, the global Lagrange stability of INNs with discrete and distributed time-varying delays [31, 32]. In [33], Huang and Cao investigated the stability of inertial CGNNs with the method of model transformation, differential mean value theorem, Lyapunov stability theory, and Linear Matrix Inequality (LMI) techniques. Maharajan et al. [34] investigated the Lagrange stability of inertial BAMNNs on the basis of Lyapunov-Krasovskiii Functional (LKF), integral inequality, and LMI approach. Tang et al. [35] proposed the exponential stability of complex-valued INNs using LKF and LMI. Kong et al. [36] studied the novel fixed-time stability of fuzzy INNs by using the inclusion theory and LKF approach.

Due to various reasons such as aging of device, existence of error in modeling, and exterior noise in the course of the hardware implementation, the parameter uncertainty occurs, which is unavoidable. Moreover, the system variables of NNs are affected by some improper dynamical responds such as instability, bifurcation, and oscillation. Recent days, many scholars did their research works in the domain of uncertain inertial neural networks [37-39].

$$
\ddot{x}(t)=-\left(a_{i}+\Delta a_{i}\right) \dot{x}_{i}(t)-\left(b_{i}+\Delta b_{i}\right) x_{i}(t)+\sum_{j=1}^{n}\left(c_{i j}+\Delta c_{i j}\right) f_{j}\left(x_{j}(t)\right)+I_{i} \text {. }
$$

Moreover, in the previous studies of INNs, most of the authors used the reduced order approach, which means that they reduced the order of the system from second order into first order by using the variable transformation. Evidently, the variable transformation leads an increase in dimension of the system and few more parameters have to be introduced. This will initiate the computational complexity of the work.

Furthermore, Lyapunov function [40] which is a scalar function defined in the phase space is an effective tool in obtaining the stability of dynamical systems. Comparing to the linear system to verify the stability of nonlinear system is a difficult task; to overcome this problem, the Lyapunov function was proposed by A. M. Lyapunov. Now a days, various kinds of Lyapunov functionals are applied in the stability of neural networks system, for instances [41] Kong et al. studied the synchronization INNs on the basis of indefinite Lyapunov-Krasovskii functional method, by utilizing the new augmented Lyapunov-Krasovskii functional. Gao et al. [42] investigated the stability problem of NNs. The stability of impulsive system is proposed by using a novel Lyapunov-like functional approach by Shao et al. [27].

From this, it is well known that, in stability theory, the Lyapunov method plays a major role, but the construction of a suitable Lyapunov function is quite harder. In order to overcome this situation, a novel technique was explored in the base of Lyapunov method and graph theory. In this approach, we have incorporated the concepts of graph theory with Lyapunov theory. In particular, in this approach, 
the topological structure of the NNs is observed as a digraph accompanied by single neuron as a node and interaction between them as a directed arcs.

Compared with the aforementioned works, the aim of this work is as listed below:

(i) In this work, we investigate the exponential stability results of inertial neural networks along with the existence of uncertainty and distributed and proportional time-varying delay.

(ii) Without using the traditional variable transformation approach, in this work, we directly design the second order system on the basis of novel Lyapunov functional which contains the first order state variable and the cofactor of the ith diagonal element of the Laplacian matrix in graph theory.

(iii) By utilizing the homeomorphism theorem, the sufficient conditions for the existence of unique equilibrium point are derived for the designed framework.

(iv) It is pointed out that the necessary criteria for the stability results are presented under the Lyapunov-Krasovskii functional and the concepts of graph theory in direct approach, which is totally different from the traditional reduced order approach.

(v) In the end of this work, we give a numerical example and simulation results to show the effectiveness of the work.

We systemized the article content in the following way: Section 2, describes the mathematical framework of the accompanied by proportional and distributed time-varying delays. Afterwards, the basic concepts on this subject are given. The solution of existence of an unique equilibrium point is discussed in Section 3, and in Section 4, the exponential stability is discussed. In Section 5, a numerical illustration is derived and the conclusion of this study is given in Section 6.

\section{Basic Concepts and Model Description}

In the present study, the existence and exponential stability of unique equilibrium point for uncertain delayed inertial neural networks (UDINN) with proportional delays are investigated.

For the entire study, we consider the following: we examine $\mathbb{M}=\{1,2, \ldots, n\}, \mathbb{R}=(-\infty,+\infty)$ represents the set of all real numbers, $\mathbb{R}^{+}=(0,+\infty), \mathbb{N}$ represents the set of all natural numbers, the $n$-dimensional Euclidean space is $\mathbb{R}^{n}$ and the set of all $n \times m$ real matrices is $\mathbb{R}^{n \times m}$, and the Euclidean norm for any vector $y$ and the trace norm for any matrix $A$ are denoted $|y|$ and $\sqrt{\operatorname{trace}\left(A^{T} A\right)}$, respectively. A digraph $\mathfrak{G}=(\mathfrak{V}, \mathfrak{F})$ is weighted, if we allocate a positive weight $m_{a b}$ for every arcs $(a, b)$. The Laplacian matrix of $\mathfrak{G}$ is defined as

$$
\mathscr{L}_{p}= \begin{cases}-m_{a b}, & \text { if } a \neq b, \\ \sum_{h \neq j} m_{a h}, & a=b .\end{cases}
$$

For the Lyapunov function, $v_{i}\left(t, y_{i}\right) \in \mathbf{C}^{1,2}$ $\left(\mathbb{R}^{+} \times \mathbb{R}^{n} ; \mathbb{R}^{+}\right), i \in \mathbb{M}$, which is differentiable and continuous at $t$ and twice differentiable at $y_{i}$.

Lemma 1 [43]). The weighted digraph $(\mathfrak{G}, \mathfrak{A})$ withn $\geq 2$ of vertices and $\mathfrak{A}=\left(a_{i j}\right)_{n \times n}$. Let the collection of all spanning unicyclic of $(\mathfrak{G}, \mathfrak{\mathfrak { U }})$ be $\mathfrak{U}$ andithdiagonal element of cofactor of the $\mathfrak{\Omega}_{p}$ is denoted $a s c_{i}$, then the following identity holds:

$$
\begin{aligned}
& \sum_{i, j=1}^{n} c_{i} a_{i} F_{i j}\left(t, z_{i}(t), z_{j}(t)\right) \\
& \quad \leq \sum_{\mathcal{U} \in \mathcal{U}} \mathscr{W}(\mathcal{U}) \sum_{(k, h) \in \mathrm{E}_{\mathfrak{G}_{\mathcal{U}}}} F_{k h}\left(t, z_{k}(t), z_{h}(t)\right),
\end{aligned}
$$

where, for $k, h \in \mathbb{M}$, an arbitrary function $F_{k h}\left(t, z_{k}(t), z_{h}(t)\right)$, the set of all spanning unicyclic graph $\mathfrak{U}, \mathscr{W}(\mathscr{U})$, and $\mathfrak{r}_{\mathscr{U}}$ denotes, respectively, the weight and dicycle of $\mathcal{U}$. Additionally, $\quad c_{i}>0$ if $(\mathfrak{G}, \mathfrak{U})$ is strongly connected for $i=1,2, \ldots, n$.

Lemma 2 (Homeomorphism theorem 44). If $\mathscr{H}$ is a continuous function from $\mathbb{R}^{n}$ to $\mathbb{R}^{n}$ which satisfies the following conditions,

(1) $\mathscr{H}(u)$ is one-one function on $\mathbb{R}^{n}$,

(2) $\|\mathscr{H}(u)\| \longrightarrow \infty$, as $\|u\| \longrightarrow \infty$,

then $\mathscr{H}(u)$ is homeomorphism of $\mathbb{R}^{n}$.

Lemma 3 (Cauchy-Schwarz inequality 45). Let $\left(\alpha_{1}, \alpha_{2}\right.$, $\left.\ldots, \alpha_{n}\right)$ and $\left(\alpha_{1}, \alpha_{2}, \ldots, \alpha_{n}\right)$ be two sequences of real numbers, one has

$$
\sum_{i=1}^{n} \alpha_{i}^{2} \sum_{i=1}^{n} \beta_{i}^{2} \geq\left(\sum_{i=1}^{n} \alpha_{i} \beta_{i}\right)^{2}
$$

the inequality turns into equality, whenever the real sequences $\left(\alpha_{1}, \alpha_{2}, \ldots, \alpha_{n}\right)$ and $\left(\alpha_{1}, \alpha_{2}, \ldots, \alpha_{n}\right)$ are proportional.

Compared with the aforementioned discussions, we describe the following uncertain neural networks with distributed, proportional delay and inertial term as

$$
\begin{aligned}
\ddot{y}_{i}(t)= & -\left(\alpha_{i}+\Delta \alpha_{i}\right) \dot{y}_{i}(t)-\left(\beta_{i}+\Delta \beta_{i}\right) y_{i}(t)+\sum_{j=1}^{n}\left(\gamma_{i j}+\Delta \gamma_{i j}\right) f_{j}\left(y_{j}(t)\right) \\
& +\sum_{j=1}^{n}\left(\delta_{i j}+\Delta \delta_{i j}\right) g_{j}\left(y_{j}\left(p_{i} t\right)\right)+\sum_{j=1}^{n}\left(\zeta_{i j}+\Delta \zeta_{i j}\right) \int_{t-\tau_{i}(t)}^{t} h_{j}\left(y_{j}(s)\right) \mathrm{d} s+I_{i},
\end{aligned}
$$


where $y_{i}(t) \in \mathbb{R}^{n}$ denotes the ith node of neurons, the positive parameters $\alpha_{i}, \beta_{i}, \gamma_{i j}, \delta_{i j}, \zeta_{i j} \in \mathbb{R}$ indicate, respectively, connection weights of ith node and the connection weight of ith node to $j$ th node. The unknown weighted matrices $\Delta \alpha_{i}, \Delta \beta_{i}, \Delta \gamma_{i j}, \Delta \delta_{i j}, \Delta \zeta_{i j}$ with the conditions $\left\|\Delta \alpha_{i}\right\| \leq \lambda_{1 i} ;\left\|\Delta \beta_{i}\right\| \leq \lambda_{2 i} ;\left\|\Delta \gamma_{i j}\right\| \leq \lambda_{3 i} ;\left\|\Delta \delta_{i j}\right\| \leq \lambda_{4 i} ;\left\|\Delta \zeta_{i j}\right\| \leq \lambda_{5 i}$. $f_{j}, g_{j}, h_{j}$ denote, respectively, the activation function, activation function of the proportional delay $p_{i} t$, and the activation function of the distributed delay $\tau_{i}(t)$ with the condition $0 \leq \dot{\tau}_{i}(t)<\tau_{i}$.

The initial value of the given system (9) is

$$
\begin{aligned}
& y_{i}(s)=\phi_{i}(s), \\
& \frac{\mathrm{d} y_{i}(s)}{\mathrm{d} s}=\psi_{i}(s), \quad-\tau \leq s \leq 0 .
\end{aligned}
$$

Remark 1. The proportional delay $p_{i} t$ is also known as unbounded time-varying delay, since the delay factor, $\left(1-p_{i}\right) t=\sigma(t)$. Here, $\sigma(t)$ is a continuous function which fulfils the condition $\sigma(t) \longrightarrow \infty$ as $t \longrightarrow \infty$. More precisely, suppose that in system (9), if $p_{i}=1$ and there is no distributed delay and also with the absence of an uncertainty, equation (9) can be rewritten as

$$
\ddot{y}_{i}(t)=-\alpha_{i} \dot{y}_{i}(t)-\beta_{i} y_{i}(t)+\sum_{j=1}^{n} \tilde{\gamma}_{i j} \tilde{f}_{j}\left(y_{j}(t)\right),
$$

which is generalized INNs.

\section{Existence of a Unique Equilibrium Point}

Definition 1. Let $y(t)=\left(y_{1}(t), y_{2}(t), \ldots, y_{n}(t)\right)$ be the solution of (9), satisfying the initial conditions. If there exist two positive constantsrand $M_{\phi, \psi}$ such that

$$
\begin{aligned}
& \left|\dot{y}_{i}(t)\right| \leq M_{\phi, \psi} e^{-r t}, \\
& \left|y_{i}(t)\right| \leq M_{\phi, \psi} e^{-r t}, \quad \forall t \in[0,+\infty),
\end{aligned}
$$

for allt $\geq t_{0}$, then the system (9) is globally exponentially stable.

Assumption 1. $\left(\mathscr{R}_{1}\right)$ For any $i=1,2, \ldots, n$, the transfer functions $f_{j}(\cdot), g_{j}(\cdot)$ and $h_{j}(\cdot)$ which is from $\mathbb{R}^{n}$ to $\mathbb{R}^{n}$ satisfies the Lipschitz condition. That is, there exist Lipschitz constants $F_{j}, G_{j}$, an $d H_{j}$ such that for any $u, v \in \mathbb{R}$,

$$
\begin{gathered}
\left|f_{j}(u)-f_{j}(v)\right| \leq F_{j}|u-v|, \\
\left|g_{j}(u)-g_{j}(v)\right| \leq G_{j}|u-v|, \\
\left|h_{j}(u)-h_{j}(v)\right| \leq H_{j}|u-v| .
\end{gathered}
$$

Assumption 2. $\left(\mathscr{R}_{2}\right)$ For each $i \in \mathbb{M}$, there exist some positive constants $a_{i}, b_{i}$, and $c_{i}$ such that

$$
\begin{aligned}
& \mathscr{A}_{i}^{r}<0, \\
& 4 \mathscr{A}_{i}^{r} \mathscr{B}_{i}^{r}>\left(\mathscr{C}_{i}^{r}\right)^{2}, \quad \forall i \in n .
\end{aligned}
$$

Assumption 3. $\left(\mathscr{R}_{3}\right)$ For each $i \in \mathbb{M}$, there exist some positive constants $a_{i}$ and $c_{i}$ such that

$$
\begin{aligned}
& a_{i}^{2}\left|\beta_{i}+\lambda_{2 i}\right|+a_{i} c_{i}\left|\alpha_{i}+\lambda_{1 i}\right|-c_{i}^{2} \\
& \mathscr{A}_{i}^{r}<0, \\
& \mathscr{B}_{i}^{r}
\end{aligned}
$$

For the sake of simplicity, we present the upcoming denotations:

$$
\begin{aligned}
\mathscr{N}_{i j}= & F_{j}\left(a_{i}^{2}\left|\gamma_{i j}+\lambda_{3 i}\right|+a_{i} c_{i}\left|\gamma_{i j}+\lambda_{3 i}\right|\right)+p_{i}\left(0 a_{i}^{2}\left|\delta_{i j}+\lambda_{i 4}\right|+\left|a_{i} c_{i}\right|\left|\delta_{i j}+\lambda_{i 4}\right|\right) e^{\left(1-p_{i}\right)} G_{j}+p_{i} \\
& \times\left(a_{i}^{2}\left|\zeta_{i j}+\lambda_{i 5}\right|+\left|a_{i} c_{i}\right|\left|\zeta_{i j}+\lambda_{i 5}\right|\right) H_{j} e^{2 r \tau_{i}} \tau_{i}, \\
\mathscr{A}_{i}^{r}= & a_{i} c_{i}-a_{i}^{2}\left|\alpha_{i}+\lambda_{1 i}\right|+\frac{1}{2} \sum_{j=1}^{n} a_{i}^{2}\left[F_{j}\left|\gamma_{i j}+\lambda_{3 i}\right|+G_{j}\left|\delta_{i j}+\lambda_{4 i}\right|+H_{j}\left|\zeta_{i j}+\lambda_{5 i}\right|\right], \\
\mathscr{B}_{i}^{r}= & -a_{i} c_{i}\left|\beta_{i}+\lambda_{2 i}\right|+\frac{1}{2} \sum_{j=1}^{n} a_{j}^{2}\left[F_{j}\left|\gamma_{i j}+\lambda_{3 i}\right|+G_{j}\left|\delta_{i j}+\lambda_{4 i}\right|+H_{j}\left|\zeta_{i j}+\lambda_{5 i}\right|\right]+\frac{1}{2} \\
& \sum_{j=1}^{n} \times\left[F_{i}\left|\gamma_{j i}+\lambda_{3 j}\right|+G_{i}\left|\delta_{j i}+\lambda_{4 j}\right|+H_{j}\left|\zeta_{j i}+\lambda_{5 j}\right|\right]\left|a_{j} c_{j}\right|+\frac{1}{2} \sum_{j=1}^{n}\left[F_{j}\left|\gamma_{i j}+\lambda_{3 i}\right|\right. \\
& \left.+G_{j}\left|\delta_{i j}+\lambda_{4 i}\right|+H_{j}\left|\zeta_{i j}+\lambda_{5 i}\right|\right]\left|a_{i} c_{i}\right|, \\
\mathscr{C}_{i}^{r}= & b_{i}+c_{i}^{2}-a_{i} c_{i}\left|\alpha_{i}+\lambda_{1 i}\right|-a_{i}^{2}\left|\beta_{i}+\lambda_{2 i}\right|,
\end{aligned}
$$


where $a_{i}, b_{i}$, and $c_{i}$ are positive constants, for all $i \in \mathbb{M}$.

Theorem 1. Suppose that there is a constant $\mathscr{B}_{i}^{r}<0$ which is defined in denotation, and assumption $\mathscr{R}_{1}$ holds, then there exists a unique equilibrium point for system (9).
Proof. Let us begin with a map $\mathscr{H}\left(y_{i}\right)=\left(\mathscr{H}_{1}\left(y_{1}\right)\right.$, $\left.\mathscr{H}_{2}\left(y_{2}\right), \ldots, \mathscr{H}_{n}\left(y_{n}\right)\right) \in \mathbb{R}^{n}$. Here,

$$
\mathscr{H}\left(y_{i}\right)=-\left(\beta_{i}+\lambda_{2 i}\right) y_{i}+\sum_{j=1}^{n}\left(\gamma_{i}+\lambda_{3 i}\right) f_{j}\left(y_{j}\right)+\sum_{j=1}^{n}\left(\delta_{i}+\lambda_{4 i}\right) g_{j}\left(y_{j}\right)+\sum_{j=1}^{n}\left(\zeta_{i}+\lambda_{5 i}\right) h_{j}\left(y_{j}\right)+I_{i} .
$$

Next, by using Lemma 2, we will show that $\mathscr{H}\left(y_{i}\right)$ is homeomorphism for all $i \in \mathbb{M}$. Initially, we show that the map $\mathscr{H}\left(y_{i}\right)$ is a one-one map on $\mathbb{R}^{n}$. Suppose if not, then there exist $u_{i}, v_{i} \in \mathbb{R}^{n}$ and $u_{i} \neq v_{i}$ such that $\mathscr{H}\left(u_{i}\right)=\mathscr{H}\left(v_{i}\right)$,

$$
\begin{aligned}
\left(\beta_{i}+\lambda_{2 i}\right)\left(u_{i}-v_{i}\right)= & \sum_{j=1}^{n}\left(\gamma_{i j}+\lambda_{3 i}\right)\left(f_{j}\left(u_{j}\right)-f_{j}\left(v_{j}\right)\right)+\sum_{j=1}^{n}\left(\delta_{i j}+\lambda_{4 i}\right)\left(g_{j}\left(u_{j}\right)-g_{j}\left(v_{j}\right)\right) \\
& +\sum_{j=1}^{n}\left(\zeta_{i j}+\lambda_{5 i}\right)\left(h_{j}\left(u_{j}\right)-h_{j}\left(v_{j}\right)\right) .
\end{aligned}
$$

Then,

$$
\begin{aligned}
\sum_{i=1}^{n} a_{i} c_{i}\left(\beta_{i}+\lambda_{2 i}\right)\left(u_{i}-v_{i}\right)^{2}= & \sum_{i=1}^{n} \sum_{j=1}^{n} a_{i} c_{i}\left(u_{i}-v_{i}\right)\left(\gamma_{i j}+\lambda_{3 i}\right)\left(f_{j}\left(u_{j}\right)-f_{j}\left(v_{j}\right)\right)+\sum_{i=1}^{n} \sum_{j=1}^{n} a_{i} c_{i} \\
& \times\left(u_{i}-v_{i}\right)\left(\delta_{i j}+\lambda_{4 i}\right)\left(g_{j}\left(u_{j}\right)-g_{j}\left(v_{j}\right)\right)+\sum_{i=1}^{n} \sum_{j=1}^{n} a_{i} c_{i}\left(u_{i}-v_{i}\right) \\
& \times\left(\zeta_{i j}+\lambda_{5 i}\right)\left(h_{j}\left(u_{j}\right)-h_{j}\left(v_{j}\right)\right) \leq \sum_{i=1}^{n} \sum_{j=1}^{n}\left|a_{i} c_{i}\right|\left(\left|\gamma_{i j}+\lambda_{3 i}\right| F_{j}+\left|\delta_{i j}+\lambda_{4 i}\right| G_{j}+\left|\zeta_{i j}+\lambda_{5 i}\right| H_{j}\right) \\
& \times\left|u_{i}-v_{i}\right|\left|u_{j}-v_{j}\right| \leq \sum_{i=1}^{n} \sum_{j=1}^{n} \frac{\left|a_{i} c_{i}\right|}{2}\left(\left|\gamma_{i j}+\lambda_{3 i}\right| F_{j}+\left|\delta_{i j}+\lambda_{4 i}\right| G_{j}+\left|\zeta_{i j}+\lambda_{5 i}\right| H_{j}\right) \\
& \cdot\left(u_{i}-v_{i}\right)^{2}+\sum_{i=1}^{n} \sum_{j=1}^{n} \frac{\left|a_{j} c_{j}\right|}{2}\left(\left|\gamma_{j i}+\lambda_{3 j}\right| F_{i}+\left|\delta_{j i}+\lambda_{4 j}\right| G_{i}\right. \\
& \left.+\left|\zeta_{j i}+\lambda_{5 j}\right| H_{i}\right)\left(u_{i}-v_{i}\right)^{2} \\
\leq & \sum_{i=1}^{n}\left\{a_{i} c_{i}\left(\beta_{i}+\lambda_{2 i}\right)-\frac{1}{2} \sum_{j=1}^{n}\left(\left|\gamma_{i j}+\lambda_{3 i}\right| F_{j}+\left|\delta_{i j}+\lambda_{4 i}\right| G_{j}\right.\right. \\
& \left.+\left|\zeta_{i j}+\lambda_{5 i}\right| H_{j}\right)\left|a_{i} c_{i}\right|-\frac{1}{2} \sum_{j=1}^{n}\left(\left|\gamma_{j i}+\lambda_{3 j}\right| F_{i}+\left|\delta_{j i}+\lambda_{4 j}\right| G_{i}\right. \\
& \left.\left.+\left|\zeta_{j i}+\lambda_{5 j}\right| H_{i}\right)\left|a_{j} c_{j}\right|\right\}\left(u_{i}-v_{i}\right)^{2}
\end{aligned}
$$


Since, $\mathscr{B}_{i}^{r}<0$, it shows that $u_{i}=v_{i}, \forall i \in \mathbb{M}$; this implies the contradiction to $u_{i} \neq v_{i}$. Hence, $\mathscr{H}\left(y_{i}\right)$ is one-one function. Our next aim is to show that $\left\|\mathscr{H}\left(y_{i}\right)\right\|_{p} \longrightarrow+\infty$ as $\left\|y_{i}\right\|_{p} \longrightarrow+\infty$.

$$
\begin{aligned}
& \sum_{i=1}^{n} y_{i} a_{i} c_{i}\left[\mathscr{H}\left(y_{i}\right)-\mathscr{H}(0)\right]=-\sum_{i=1}^{n}\left(\beta_{i}+\lambda_{2 i}\right) a_{i} c_{i} y_{i}^{2}+\sum_{i=1}^{n} \sum_{j=1}^{n} a_{i} c_{i}\left(\gamma_{i j}+\lambda_{3 i}\right) y_{i}\left(f_{j}\left(y_{j}\right)-f_{j}(0)\right) \\
& \quad+\sum_{i=1}^{n} \sum_{j=1}^{n} a_{i} c_{i}\left(\delta_{i j}+\lambda_{4 i}\right) y_{i}\left(g_{j}\left(y_{j}\right)-g_{j}(0)\right)+\sum_{i=1}^{n} \sum_{j=1}^{n} a_{i} c_{i}\left(\zeta_{i j}\right. \\
& \left.+\lambda_{5 i}\right) y_{i}\left(h_{j}\left(y_{j}\right)-h_{j}(0)\right) \leq-\sum_{i=1}^{n}\left[\left(\beta_{i}+\lambda_{2 i}\right) a_{i} c_{i} y_{i}^{2}-\sum_{j=1}^{n} a_{i} c_{i}\left(\gamma_{i j}+\lambda_{3 i}\right) y_{i} F_{j} y_{j}-\sum_{j=1}^{n} a_{i} c_{i}\left(\delta_{i j}\right.\right. \\
& \left.\left.\quad+\lambda_{4 i}\right) y_{i} G_{j} y_{j}-\sum_{j=1}^{n} a_{i} c_{i}\left(\zeta_{i j}+\lambda_{5 i}\right) y_{i} H_{j} y_{j}\right] \\
& \leq-\sum_{i=1}^{n}\left[a_{i} c_{i}\left(\beta_{i}+\lambda_{2 i}\right)-\frac{1}{2} \sum_{j=1}^{n}\left(\left|\gamma_{i j}+\lambda_{3 i}\right| F_{j}+\left|\delta_{i j}+\lambda_{4 i}\right| G_{j}+\mid \zeta_{i j}\right.\right. \\
& \left.\quad+\lambda_{5 i} \mid H_{j}\right)\left|a_{i} c_{i}\right|-\frac{1}{2} \sum_{j=1}^{n}\left(\left|\gamma_{j i}+\lambda_{3 j}\right| F_{i}+\left|\delta_{j i}+\lambda_{4 j}\right| G_{i}+\left|\zeta_{j i}+\lambda_{5 j}\right|\right. \\
& \left.\left.\quad \times H_{i}\right)\left|a_{j} c_{j}\right|\right] y_{i}^{2} \\
& \leq-\xi_{i} \sum_{i=1}^{n} y_{i}^{2} .
\end{aligned}
$$

Here,

$$
\begin{aligned}
\xi_{i}= & \min _{1 \leq i \leq n}\left\{a_{i} c_{i}\left(\beta_{i}+\lambda_{2 i}\right)-\frac{1}{2} \sum_{j=1}^{n}\left(\left|\gamma_{i j}+\lambda_{3 i}\right| F_{j}+\left|\delta_{i j}+\lambda_{4 i}\right| G_{j}+\left|\zeta_{i j}+\lambda_{5 i}\right| H_{j}\right)\left|a_{i} c_{i}\right|-\frac{1}{2}\right. \\
& \left.\times \sum_{j=1}^{n}\left(\left|\gamma_{j i}+\lambda_{3 j}\right| F_{i}+\left|\delta_{j i}+\lambda_{4 j}\right| G_{i}+\left|\zeta_{j i}+\lambda_{5 j}\right| H_{i}\right)\left|a_{j} c_{j}\right|\right\},
\end{aligned}
$$

and $\xi_{i}>0$, since $\mathscr{B}_{i}^{r}<0, \forall i \in \mathbb{M}$.

$$
\sum_{i=1}^{n} y_{i} a_{i} c_{i}\left[\mathscr{H}\left(y_{i}\right)-\mathscr{H}(0)\right] \leq-\xi_{i} \sum_{i=1}^{n} y_{i}^{2} .
$$

By using Cauchy-Schwartz inequality in Lemma 3,

$$
\begin{aligned}
& \sum_{i=1}^{n} y_{i}^{2} \leq \frac{1}{\xi_{i}} \sum_{i=1}^{n}\left|a_{i} c_{i}\right|\left|y_{i}\right|\left|\mathscr{H}\left(y_{i}\right)-\mathscr{H}(0)\right| \\
& \leq \frac{a}{\xi_{i}} \sum_{i=1}^{n}\left|y_{i}\right|\left|\mathscr{H}\left(y_{i}\right)-\mathscr{H}(0)\right| .
\end{aligned}
$$

where $a=\max _{1 \leq i \leq n}\left\{\left|a_{i} c_{i}\right|\right\}$. It follows that

$$
\|y\|_{2} \leq \frac{a}{\xi}\left\|\mathscr{H}_{i}(y)-\mathscr{H}(0)\right\|_{2} \leq \frac{a}{\xi}\left(\left\|\mathscr{H}_{i}(y)\right\|_{2}-\|\mathscr{H}(0)\|_{2}\right),
$$

which reveals that, $\left\|\mathscr{H}\left(y_{i}\right)\right\|_{2} \longrightarrow+\infty$ as $\left\|y_{i}\right\| \longrightarrow+\infty$. By Lemma $2, \mathscr{H}\left(y_{i}\right)$ is a homeomorphism on $\mathbb{R}^{n}$ which implies that system (9) has a unique equilibrium point $y_{i}^{*}=\left(y_{1}^{*}, y_{2}^{*}\right.$, $\left.\ldots, y_{n}^{*}\right)^{T}$.

Corollary 1. Suppose that the assumptions $\mathscr{R}_{1}$ and $\mathscr{R}_{3}$ hold, then (9) has a unique equilibrium point, which is asymptotically stable. 
Remark 2. Suppose that the value of uncertain parameters is equal to zero; there occurs a change of an unbounded timevarying delay (proportional delay) into discrete time-varying delay, and then system (9) is converted into INNs with discrete and distributed model and it is described as follows:

$$
\ddot{y}_{i}(t)=-\alpha_{i} \dot{y}_{i}(t)-\beta_{i} y_{i}(t)+\sum_{j=1}^{n} \gamma_{i j} f_{j}\left(y_{j}(t)\right)+\sum_{j=1}^{n} \delta_{i j} g_{j}\left(y_{j}(t-\eta(t))\right)+\sum_{j=1}^{n} \zeta_{i j} \int_{t-\tau_{i}(t)}^{t} h_{j}\left(y_{j}(s)\right) \times \mathrm{d} s+I_{i}
$$

\section{Exponential Stability}

Theorem 2. Under the assumptions $\left(\mathscr{R}_{1}\right)$ and $\left(\mathscr{R}_{2}\right)$, the system (9) is exponentially stable. Proof. Let us defined the global Lyapunov functional as follows

$$
V(t)=\sum_{i=1}^{n} \varsigma_{i} v_{i}(t)
$$

Where, $\varsigma_{i}$ denotes the cofactor of ith diagonal element of $\mathscr{L}_{p}$ of digraph $(\mathscr{G}, \mathscr{A})$ and

$$
v_{i}(t)=v_{i}^{(1)}(t)+v_{i}^{(2)}(t)+v_{i}^{(3)}(t)+v_{i}^{(4)}(t) .
$$

Here,

$$
\begin{aligned}
& v_{i}^{(1)}(t)=\frac{1}{2} \sum_{i=1}^{n} b_{i} y_{i}^{2}(t) e^{2 r t} \\
& v_{i}^{(2)}(t)=\frac{1}{2} \sum_{i=1}^{n}\left(a_{i} \dot{y}_{i}(t)+c_{i} y_{i}(t)\right)^{2} e^{2 r t} \\
& v_{i}^{(3)}(t)=\frac{1}{2} \sum_{i=1}^{n} \sum_{j=1}^{n} \frac{1}{p_{i} G_{j}}\left(a_{i}^{2}\left|\delta_{i j}+\lambda_{i 4}\right|+\left|a_{i} c_{i}\right|\left|\delta_{i j}+\lambda_{i 4}\right|\right) e^{\left(1-p_{i}\right)} \int_{p_{i} t}^{t} e^{2 r s} f^{2}\left(y_{j}(s)\right) \mathrm{d} s, \\
& v_{i}^{(4)}(t)=\frac{1}{2} \sum_{i=1}^{n} \sum_{j=1}^{n} \frac{1}{H_{j}}\left(a_{i}^{2}\left|\zeta_{i j}+\lambda_{i 5}\right|+\left|a_{i} c_{i}\right|\left|\zeta_{i j}+\lambda_{i 5}\right|\right) e^{2 r \tau_{i}} \int_{-\tau_{i}(t)}^{0} \int_{t+\theta}^{\theta} e^{2 r s} f^{2}\left(y_{j}(s)\right) \mathrm{d} s \mathrm{~d} \theta .
\end{aligned}
$$

Along the solution (9) we derive $v_{i}(t)$ as follows

$$
\begin{aligned}
\dot{v}_{i}^{(1)}(t)= & \frac{1}{2} \sum_{i=1}^{n} b_{i} e^{2 r t}\left[2 \dot{y}_{i}(t) y_{i}(t)+2 r y_{i}^{2}(t)\right]=\sum_{i=1}^{n} b_{i} e^{2 r t} \dot{y}_{i}(t) y_{i}(t)+\frac{1}{2} \sum_{i=1}^{n} 2 r b_{i} e^{2 r t} y_{i}^{2}(t), \\
\dot{v}_{i}^{(2)}(t)= & \frac{1}{2} \sum_{i=1}^{n}\left[2 r e^{2 r t}\left(a_{i} \dot{y}_{i}(t)+c_{i} y_{i}(t)\right)^{2}+2 e^{2 r t}\left(a_{i} \dot{y}_{i}(t)+c_{i} y_{i}(t)\right)\left(a_{i} \ddot{y}_{i}(t)+c_{i} \dot{y}_{i}(t)\right)\right] \\
= & \frac{1}{2} \sum_{i=1}^{n} e^{2 r t}\left(2 r a_{i}^{2}+2 a_{i} c_{i}\right) \dot{y}_{i}^{2}(t)+\frac{1}{2} \sum_{i=1}^{n} e^{2 r t}\left(2 a_{i}^{2} \dot{y}_{i}(t)+2 a_{i} c_{i} y_{i}(t)\right) \ddot{y}_{i}(t)+\frac{1}{2} \sum_{i=1}^{n} e^{2 r t} \\
& \times\left(4 r a_{i} c_{i}+2 c_{i}^{2}\right) y_{i}(t) \dot{y}_{i}(t)+\frac{1}{2} \sum_{i=1}^{n} e^{2 r t} 2 r c_{i}^{2} y_{i}^{2}(t),
\end{aligned}
$$




$$
\begin{aligned}
\dot{v}_{i}^{(3)}(t)= & \frac{1}{2} \sum_{i=1}^{n} \sum_{j=1}^{n} \frac{1}{p_{i}}\left(a_{i}^{2}\left|\delta_{i j}+\lambda_{i 4}\right|+\left|a_{i} c_{i}\right|\left|\delta_{i j}+\lambda_{i 4}\right|\right) e^{\left(1-p_{i}\right)} G_{j}\left[e^{2 r t} y_{j}^{2}(t)-e^{2 r p_{i} t} y_{j}^{2}\left(p_{i} t\right) p_{i}\right] \\
= & \frac{1}{2} \sum_{i=1}^{n} \sum_{j=1}^{n} e^{2 r t} G_{j}\left[\frac{1}{p_{i}}\left(a_{i}^{2}\left|\delta_{i j}+\lambda_{i 4}\right|+\left|a_{i} c_{i}\right|\left|\delta_{i j}+\lambda_{i 4}\right|\right) e^{\left(1-p_{i}\right)} y_{j}^{2}(t)-\left(a_{i}^{2}\left|\delta_{i j}+\lambda_{i 4}\right|\right)\right. \\
& \left.\left.+\left|a_{i} c_{i}\right|\left|\delta_{i j}+\lambda_{i 4}\right|\right) y_{j}^{2}\left(p_{i} t\right)\right], \\
\dot{v}_{i}^{(4)}(t)= & \frac{1}{2} \sum_{i=1}^{n} \sum_{j=1}^{n}\left(a_{i}^{2}\left|\zeta_{i j}+\lambda_{i 5}\right|+\left|a_{i} c_{i}\right|\left|\zeta_{i j}+\lambda_{i 5}\right|\right) H_{j}\left[e^{2 r\left(\tau_{i}+t\right)} \tau_{i} y_{j}^{2}(t)-e^{2 r \tau_{i}} \int_{t-\tau_{i}(t)}^{t} e^{2 r t} y_{j}^{2}(s) \mathrm{d} s\right] .
\end{aligned}
$$

Substitute (29)-(32) in (27) we get,

$$
\begin{aligned}
& \dot{v}_{i}(t)= \sum_{i=1}^{n} e^{2 r t}\left\{\left(b_{i}+2 r a_{i} c_{i}+c_{i}^{2}\right) \dot{y}_{i}(t) y_{i}(t)+\left(r a_{i}^{2}+a_{i} c_{i}\right) \dot{y}_{i}^{2}(t)+\left(r b_{i}+r c_{i}^{2}\right) y_{i}^{2}(t)+\left(2 a_{i}^{2}\right.\right. \\
&\left.\times \dot{y}_{i}(t)+2 a_{i} c_{i} y_{i}(t)\right) y_{i}(t)+\frac{1}{2} \sum_{j=1}^{n}\left[\frac{1}{p_{i}}\left(a_{i}^{2}\left|\delta_{i j}+\lambda_{i 4}\right|+\left|a_{i} c_{i}\right|\left|\delta_{i j}+\lambda_{i 4}\right|\right) e^{\left(1-p_{i}\right)} G_{j}+\left(a_{i}^{2}\right.\right. \\
&\left.\left.\times\left|\zeta_{i j}+\lambda_{i 5}\right|+\left|a_{i} c_{i}\right|\left|\zeta_{i j}+\lambda_{i 5}\right|\right) H_{j} e^{2 r \tau_{i}} \tau_{i}\right] y_{j}^{2}(t)-\frac{1}{2} \sum_{j=1}^{n}\left(a_{i}^{2}\left|\delta_{i j}+\lambda_{i 4}\right|+\left|a_{i} c_{i}\right|\left|\delta_{i j}+\lambda_{i 4}\right|\right) \\
&\left.\times G_{j} y_{i}^{2}\left(p_{i} t\right)\right\}-\frac{1}{2} \sum_{i=1}^{n} \sum_{j=1}^{n}\left(a_{i}^{2}\left|\zeta_{i j}+\lambda_{i 5}\right|+\left|a_{i} c_{i}\right|\left|\zeta_{i j}+\lambda_{i 5}\right|\right) H_{j} e^{2 r \tau_{i}} \int_{t-\tau_{i}(t)}^{t} e^{2 r t} y_{j}^{2}(s) \mathrm{d} s \\
& \leq \sum_{i=1}^{n} e^{2 r t}\left\{\left(b_{i}+2 r a_{i} c_{i}+c_{i}^{2}\right) \dot{y}_{i}(t) y_{i}(t)+\left(r a_{i}^{2}+a_{i} c_{i}\right) \dot{y}_{i}^{2}(t)+\left(r b_{i}+r c_{i}^{2}\right) y_{i}^{2}(t)-a_{i}^{2}\right. \\
&\left|\alpha_{i}+\lambda_{1 i}\right| \dot{y}_{i}^{2}(t)-a_{i} c_{i}\left|\alpha_{i}+\lambda_{1 i}\right| \dot{y}_{i}(t) y_{i}(t)-2 a_{i}^{2}\left|\beta_{i}+\lambda_{2 i}\right| \dot{y}_{i}(t) y_{i}(t)-2 a_{i} c_{i}\left|\beta_{i}+\lambda_{2 i}\right| y_{i}^{2}(t) \\
&+\sum_{j=1}^{n}\left(a_{i}^{2} \dot{y}_{i}(t)+a_{i} c_{i} y_{i}(t)\right)\left(\gamma_{i j}+\Delta \gamma_{i j}\right) f_{j}\left(y_{j}(t)\right)+\sum_{j=1}^{n}\left(a_{i}^{2} \dot{y}_{i}(t)+a_{i} c_{i} y_{i}\right)\left(\delta_{i j}+\Delta \delta_{i j}\right) \\
&\left.\times\left|\zeta_{i j}+\lambda_{i 5}\right|\right) H_{j} e^{2 r \tau_{i}} \int_{t-\tau_{i}(t)}^{t} e^{2 r t} y_{j}^{2}(s) \mathrm{d} s . \\
& \times g_{j}\left(y_{j}\left(p_{i} t\right)\right)+\sum_{j=1}^{n}\left(a_{i}^{2} \dot{y}_{i}(t)+a_{i} c_{i} y_{i}(t)\right)\left(\zeta_{i j}+\Delta \zeta_{i j}\right) \int_{t-\tau_{i}(t)}^{t} h_{j}\left(y_{j}(s)\right) \mathrm{d} s+\frac{1}{2} \sum_{j=1}^{n}\left[\frac { 1 } { p _ { i } } \left(a_{i}^{2}\right.\right. \\
&\left.\left.\times\left|\delta_{i j}+\lambda_{i 4}\right|+\left|a_{i} c_{i}\right|\left|\delta_{i j}+\lambda_{i 4}\right|\right) e^{\left(1-p_{i}\right)} G_{j}+\left(a_{i}^{2}\left|\zeta_{i j}+\lambda_{i 5}\right|+\left|a_{i} c_{i}\right|\left|\zeta_{i j}+\lambda_{i 5}\right|\right) H_{j} e^{2 r \tau_{i}} \tau_{i}\right] \\
&\left.\sum_{j=1}^{n}\left(a_{i}^{2}\left|\delta_{i j}+\lambda_{i 4}\right|+\left|a_{i} c_{i}\right|\left|\delta_{i j}+\lambda_{i 4}\right|\right) G_{j} y_{i}^{2}\left(p_{i} t\right)\right\}-\frac{1}{2} \sum_{i=1}^{n} \sum_{j=1}^{n}\left(a_{i}^{2}\left|\zeta_{i j}+\lambda_{i 5}\right|+\left|a_{i} c_{i}\right|\right. \\
& \\
& \\
&
\end{aligned}
$$

By using the assumption $\mathscr{R}_{1}$ and the inequality $u v \leq(1 / 2)\left(u^{2}+v^{2}\right)$ we get, 
Mathematical Problems in Engineering

9

$$
\begin{aligned}
& \left(a_{i}^{2} \dot{y}_{i}(t)+a_{i} c_{i} y_{i}(t)\right)\left(\gamma_{i j}+\Delta \gamma_{i j}\right) f_{j}\left(y_{j}(t)\right)=a_{i}^{2}\left(\gamma_{i j}+\Delta \gamma_{i j}\right) \dot{y}_{i}(t) f_{j}\left(y_{j}(t)\right)+a_{i} c_{i}\left(\gamma_{i j}+\Delta \gamma_{i j}\right) y_{i}(t) \times f_{j}\left(y_{j}(t)\right) \\
& \quad \leq \frac{1}{2} F_{j}\left(\gamma_{i j}+\lambda_{3 i}\right)\left(a_{i}^{2}\left(\dot{y}_{i}^{2}(t)+y_{j}^{2}(t)\right)+a_{i} c_{i}\left(y_{i}^{2}(t)+y_{j}^{2}(t)\right)\right.
\end{aligned}
$$

Similarly,

$$
\begin{aligned}
& \left(a_{i}^{2} \dot{y}_{i}(t)+a_{i} c_{i} y_{i}(t)\right)\left(\delta_{i j}+\Delta \delta_{i j}\right) g_{j}\left(y_{j}\left(p_{i} t\right)\right)=a_{i}^{2}\left(\delta_{i j}+\Delta \delta_{i j}\right) \dot{y}_{i}(t) g_{j}\left(y_{j}\left(p_{i} t\right)\right)+a_{i} c_{i}\left(\delta_{i j}+\Delta \delta_{i j}\right) \\
& \quad \times y_{i}(t) g_{j}\left(y_{j}(t)\right) \\
& \leq \frac{1}{2} G_{j}\left(\delta_{i j}+\lambda_{4 i}\right)\left(a_{i}^{2}\left(\dot{y}_{i}^{2}(t)+y_{j}^{2}\left(p_{i} t\right)\right)+a_{i} c_{i}\left(y_{i}^{2}(t)\right.\right. \\
& \left.\left.\quad+y_{j}^{2}\left(p_{i} t\right)\right)\right),
\end{aligned}
$$

and

$$
\begin{aligned}
& \left(a_{i}^{2} \dot{y}_{i}(t)+a_{i} c_{i} y_{i}(t)\right)\left(\zeta_{i j}+\Delta \zeta_{i j}\right) \int_{t-\tau_{i}(t)}^{t} h_{j}\left(y_{j}(s)\right) \mathrm{d} s=a_{i}^{2}\left(\zeta_{i j}+\Delta \zeta_{i j}\right) \dot{y}_{i}(t) \int_{t-\tau_{i}(t)}^{t} h_{j}\left(y_{j}(s)\right) d s+a_{i} c_{i} \\
& \quad \times\left(\zeta_{i j}+\Delta \zeta_{i j}\right) y_{i}(t) \int_{t-\tau_{i}(t)}^{t} h_{j}\left(y_{j}(s)\right) \mathrm{d} s \\
& \leq \frac{1}{2} H_{j}\left(\zeta_{i j}+\lambda_{5 i}\right)\left(a_{i}^{2}\left(\dot{y}_{i}^{2}(t)+\int_{t-\tau_{i}(t)}^{t}\left(y_{j}^{2}(s)\right) \mathrm{d} s\right)\right. \\
& \left.\quad+a_{i} c_{i}\left(y_{i}^{2}(t)+\int_{t-\tau_{i}(t)}^{t}\left(y_{j}^{2}(s)\right) \mathrm{d} s\right)\right) .
\end{aligned}
$$

Substitute in (34)-(36) in (33), we have, 


$$
\begin{aligned}
\dot{v}_{i}(t) \leq & \sum_{i=1}^{n} e^{2 r t}\left\{\left(b_{i}+2 r a_{i} c_{i}+c_{i}^{2}-a_{i} c_{i}\left(\alpha_{i}+\lambda_{1 i}\right)-2 a_{i}^{2}\left(\beta_{i}+\lambda_{2 i}\right)\right) \dot{y}_{i}(t) y_{i}(t)+\left(r a_{i}^{2}+a_{i} c_{i}\right.\right. \\
& \left.-a_{i}^{2}\left(\alpha_{i}+\lambda_{1 i}\right)+\sum_{j=1}^{n} \frac{F_{j}\left(\gamma_{i j}+\lambda_{3 i}\right)}{2} a_{i}^{2}+\sum_{j=1}^{n} \frac{G_{j}\left(\delta_{i j}+\lambda_{4 i}\right)}{2} a_{i}^{2}+\sum_{j=1}^{n} \frac{H_{j}\left(\zeta_{i j}+\lambda_{5 i}\right)}{2} a_{i}^{2}\right) \\
& \times \dot{y}_{i}^{2}(t)+\left(r b_{i}+r c_{i}^{2}-2 a_{i} c_{i}\left(\beta_{i}+\lambda_{2 i}\right)+\sum_{j=1}^{n} \frac{F_{j}\left(\gamma_{i j}+\lambda_{3 i}\right)}{2} a_{i} c_{i}+\sum_{j=1}^{n} \frac{G_{j}\left(\delta_{i j}+\lambda_{4 i}\right)}{2}\right. \\
& \left.\times a_{i} c_{i}+\sum_{j=1}^{n} \frac{H_{j}\left(\zeta_{i j}+\lambda_{5 i}\right)}{2} a_{i} c_{i}\right) y_{i}^{2}(t)+\frac{1}{2} \sum_{j=1}^{n}\left[\frac{1}{p_{i}}\left(a_{i}^{2}\left|\delta_{i j}+\lambda_{i 4}\right|+\left|a_{i} c_{i}\right|\left|\delta_{i j}+\lambda_{i 4}\right|\right) e^{\left(1-p_{i}\right)}\right. \\
& \times G_{j}+\left(a_{i}^{2}\left|\zeta_{i j}+\lambda_{i 5}\right|+\left|a_{i} c_{i}\right|\left|\zeta_{i j}+\lambda_{i 5}\right|\right) H_{j} e^{2 r \tau_{i}} \tau_{i}+\sum_{j=1}^{n} \frac{F_{j}\left(\gamma_{i j}+\lambda_{3 i}\right)}{2} a_{i}^{2}+\sum_{j=1}^{n} \frac{F_{j}\left(\gamma_{i j}+\lambda_{3 i}\right)}{2} \\
& \left.\times a_{i} c_{i}\right] y_{j}^{2}(t)-\frac{1}{2} \sum_{j=1}^{n}\left(a_{i}^{2}\left|\delta_{i j}+\lambda_{i 4}\right|+\left|a_{i} c_{i}\right|\left|\delta_{i j}+\lambda_{i 4}\right|\right) G_{j} y_{j}^{2}\left(p_{i} t\right)+\sum_{j=1}^{n} \frac{G_{j}\left(\delta_{i j}+\lambda_{4 i}\right)}{2} a_{i}^{2} \\
& \left.\times y_{j}^{2}\left(p_{i} t\right)+\frac{1}{2} \sum_{j=1}^{n} G_{j}\left(\delta_{i j}+\lambda_{4 i}\right) a_{i} c_{i} y_{j}\left(p_{i} t\right)\right\}-\frac{1}{2} \sum_{i=1}^{n} \sum_{j=1}^{n}\left(a_{i}^{2}\left|\zeta_{i j}+\lambda_{i 5}\right|+\left|a_{i} c_{i}\right|\left|\zeta_{i j}+\lambda_{i 5}\right|\right) \\
& \times H_{j} e^{2 r \tau_{i}} \int_{t-\tau_{i}(t)}^{t} e^{2 r t} y_{j}^{2}(s) \mathrm{d} s+\sum_{j=1}^{n} \frac{H_{j}\left(\zeta_{i j}+\lambda_{5 i}\right)}{2} a_{i}^{2} \int_{t-\tau_{i}(t)}^{t} y_{j}^{2}(s) \mathrm{d} s+\sum_{j=1}^{n} \frac{H_{j}\left(\zeta_{i j}+\lambda_{5 i}\right)}{2} a_{i} c_{i} \times \int_{t-\tau_{i}(t)}^{t} y_{j}^{2}(s) \mathrm{d} s
\end{aligned}
$$$$
\dot{v}_{i}(t) \leq \sum_{i=1}^{n} e^{2 r t}\left\{\left(b_{i}+2 r a_{i} c_{i}+c_{i}^{2}-a_{i} c_{i}\left(\alpha_{i}+\lambda_{1 i}\right)-2 a_{i}^{2}\left(\beta_{i}+\lambda_{2 i}\right)\right) \dot{y}_{i}(t) y_{i}(t)+\left(r a_{i}^{2}+a_{i} c_{i}\right.\right.
$$$$
\left.-a_{i}^{2}\left(\alpha_{i}+\lambda_{1 i}\right)+\sum_{j=1}^{n} \frac{F_{j}\left(\gamma_{i j}+\lambda_{3 i}\right)}{2} a_{i}^{2}+\sum_{j=1}^{n} \frac{G_{j}\left(\delta_{i j}+\lambda_{4 i}\right)}{2} a_{i}^{2}+\sum_{j=1}^{n} \frac{H_{j}\left(\zeta_{i j}+\lambda_{5 i}\right)}{2} a_{i}^{2}\right) \dot{y}_{i}^{2}(t)
$$$$
+\left(r b_{i}+r c_{i}^{2}-2 a_{i} c_{i}\left(\beta_{i}+\lambda_{2 i}\right)+\sum_{j=1}^{n} \frac{F_{j}\left(\gamma_{i j}+\lambda_{3 i}\right)}{2} a_{i} c_{i}+\sum_{j=1}^{n} \frac{G_{j}\left(\delta_{i j}+\lambda_{4 i}\right)}{2} a_{i} c_{i}\right.
$$$$
\left.+\sum_{j=1}^{n} \frac{H_{j}\left(\zeta_{i j}+\lambda_{5 i}\right)}{2} a_{i} c_{i}\right) y_{i}^{2}(t)+\frac{1}{2} \sum_{j=1}^{n}\left[\frac{1}{p_{i}}\left(a_{i}^{2}\left|\delta_{i j}+\lambda_{i 4}\right|+\left|a_{i} c_{i}\right|\left|\delta_{i j}+\lambda_{i 4}\right|\right) e^{\left(1-p_{i}\right)} G_{j}\right.
$$$$
\left.+\left(a_{i}^{2}\left|\zeta_{i j}+\lambda_{i 5}\right|+\left|a_{i} c_{i}\right|\left|\zeta_{i j}+\lambda_{i 5}\right|\right) H_{j} e^{2 r \tau_{i}} \tau_{i}+\frac{1}{2} \sum_{j=1}^{n} F_{j}\left(\gamma_{i j}+\lambda_{3 i}\right)\left(a_{i}^{2}+a_{i} c_{i}\right)\right] y_{j}^{2}(t)
$$

$$
\dot{v}_{i}(t) \leq \sum_{i=1}^{n} e^{2 r t}\left\{\left(b_{i}+2 r a_{i} c_{i}+c_{i}^{2}-a_{i} c_{i}\left(\alpha_{i}+\lambda_{1 i}\right)-2 a_{i}^{2}\left(\beta_{i}+\lambda_{2 i}\right)\right) \dot{y}_{i}(t) y_{i}(t)+\left(r a_{i}^{2}+a_{i} c_{i}\right.\right.
$$

$$
\begin{aligned}
& \left.-a_{i}^{2}\left(\alpha_{i}+\lambda_{1 i}\right)+\sum_{j=1}^{n} \frac{F_{j}\left(\gamma_{i j}+\lambda_{3 i}\right)}{2} a_{i}^{2}+\sum_{j=1}^{n} \frac{G_{j}\left(\delta_{i j}+\lambda_{4 i}\right)}{2} a_{i}^{2}+\sum_{j=1}^{n} \frac{H_{j}\left(\zeta_{i j}+\lambda_{5 i}\right)}{2} a_{i}^{2}\right] \dot{y}_{i}^{2}(t) \\
& +\left(r b_{i}+r c_{i}^{2}-2 a_{i} c_{i}\left(\beta_{i}+\lambda_{2 i}\right)+\sum_{j=1}^{n} \frac{F_{j}\left(\gamma_{i j}+\lambda_{3 i}\right)}{2} a_{i} c_{i}+\sum_{j=1}^{n} \frac{G_{j}\left(\delta_{i j}+\lambda_{4 i}\right)}{2} a_{i} c_{i}+\right. \\
& \left.+\sum_{j=1}^{n} \times \frac{H_{j}\left(\zeta_{i j}+\lambda_{5 i}\right)}{2} a_{i} c_{i}\right) y_{i}^{2}(t)+\frac{1}{2} \sum_{j=1}^{n} \frac{1}{p_{i}}\left\{\left[p_{i} F_{j}\left(a_{i}^{2}\left|\gamma_{i j}+\lambda_{3 i}\right|+a_{i} c_{i}\left|\gamma_{i j}+\lambda_{3 i}\right|\right)+\left(a_{i}^{2}\left|\delta_{i j}+\lambda_{i 4}\right|\right.\right.\right. \\
& \left.\left.+\left|a_{i} c_{i}\right|\left|\delta_{i j}+\lambda_{i 4}\right|\right) e^{\left(1-p_{i}\right)} G_{j}+p_{i}\left(a_{i}^{2}\left|\zeta_{i j}+\lambda_{i 5}\right|+\left|a_{i} c_{i}\right|\left|\zeta_{i j}+\lambda_{i 5}\right|\right) H_{j} e^{2 r \tau_{i}} \tau_{i}\right] y_{j}^{2}(t)-\frac{1}{2} \sum_{i=1}^{n} \frac{1}{p_{j}} \\
& \times\left[p_{j} F_{i}\left(a_{j}^{2}\left|\gamma_{j i}+\lambda_{3 j}\right|+a_{j} c_{j}\left|\gamma_{j i}+\lambda_{3 j}\right|\right)+\left(a_{j}^{2}\left|\delta_{j i}+\lambda_{j 4}\right|+\left|a_{j} c_{j}\right|\left|\delta_{j i}+\lambda_{j 4}\right|\right) e^{\left(1-p_{j}\right)} G_{i}\right. \\
& \left.\left.+p_{j}\left(a_{j}^{2}\left|\zeta_{j i}+\lambda_{j 5}\right|+\left|a_{i} c_{i}\right|\left|\zeta_{j i}+\lambda_{j 5}\right|\right) H_{i} e^{2 r \tau_{j}} \tau_{j}\right] y_{i}^{2}(t)\right\}, \\
& =e^{2 r t}\left\{\sum_{i=1}^{n}\left(\mathscr{A}_{i}^{r} \dot{y}_{i}^{2}(t)+\mathscr{C}_{i}^{r} y_{i}(t) \dot{y}_{i}(t)+\mathscr{B}_{i}^{r} y_{i}^{2}(t)+\sum_{i=1}^{n} \sum_{j=1}^{n} \mathcal{N}_{i j}\left(y_{j}^{2}(t)-y_{i}^{2}(t)\right)\right)\right\} .
\end{aligned}
$$


Here, we need to show that, $(1 / 2) \sum_{i=1}^{n} \sum_{j=1}^{n} \mathcal{N}_{i j}\left(y_{j}^{2}(t)-y_{i}^{2}(t)\right) \leq 0$. The obtained results has shown under Lemma 1 , that

$$
\frac{1}{2} \sum_{i=1}^{n} \sum_{j=1}^{n} \mathcal{N}_{i j}\left(y_{j}^{2}(t)-y_{i}^{2}(t)\right)=\frac{1}{2} \sum_{Q \in \mathbb{Q}} W(Q) \sum_{(s, r) \in E\left(C_{Q}\right)}\left(y_{r}^{2}(t)-y_{s}^{2}(t)\right) .
$$
by,

Along the directed cycle $C_{Q}$, we indicate the set $E\left(C_{Q}\right)$

$$
E\left(C_{Q}\right)=\left\{\left(i_{m}, i_{m+1}\right) \mid m=1,2, \ldots, n-1, i_{n}=i_{1}\right\} .
$$

According to (40), we get

$$
\sum_{(s, r) \in E\left(C_{Q}\right)}\left(y_{r}^{2}(t)-y_{s}^{2}(t)\right)=y_{i_{1}}^{2}(t)-y_{i_{2}}^{2}(t)+y_{i_{2}}^{2}(t)-y_{i_{3}}^{2}(t)+\cdots+y_{i_{n-1}}^{2}(t)-y_{i_{n}}^{2}(t)+y_{i_{n}}^{2}(t)-y_{i_{1}}^{2}(t)=0 .
$$

From (39) and (41) we get,

Then, by (38) implies that,

$$
\frac{1}{2} \sum_{i=1}^{n} \sum_{j=1}^{n} \mathcal{N}_{i j}\left(y_{j}^{2}(t)-y_{i}^{2}(t)\right) \leq 0
$$

$$
\begin{aligned}
\dot{v}_{i} & \leq e^{2 r t}\left\{\sum_{i=1}^{n}\left(\mathscr{A}_{i}^{r} \dot{y}_{i}^{2}(t)+\mathscr{C}_{i}^{r} y_{i}(t) \dot{y}_{i}(t)+\mathscr{B}_{i}^{r} y_{i}^{2}(t)\right)\right\} \\
& =e^{2 r t}\left\{\sum_{i=1}^{n} \mathscr{A}_{i}^{r}\left(\dot{y}_{i}^{2}(t)+\frac{\mathscr{C}_{i}^{r}}{\mathscr{A}_{i}^{r}} y_{i}(t) \dot{y}_{i}(t)\right)+\mathscr{B}_{i}^{r} y_{i}^{2}(t)\right\} \\
& =e^{2 r t}\left\{\sum_{i=1}^{n} \mathscr{A}_{i}^{r}\left(\dot{y}_{i}^{2}(t)+2 \frac{\mathscr{C}_{i}^{r}}{2 \mathscr{A}_{i}^{r}} y_{i}(t) \dot{y}_{i}(t)+\left(\frac{\mathscr{C}_{i}^{r}}{2 \mathscr{A}_{i}^{r}}\right)^{2} y_{i}^{2}(t)\right)+\left(\mathscr{B}_{i}^{r}-\frac{\left(\mathscr{C}_{i}^{r}\right)^{2}}{4\left(\mathscr{A}_{i}^{r}\right)^{2}}\right) y_{i}^{2}(t)\right\} \\
& =e^{2 r t}\left\{\sum_{i=1}^{n} \mathscr{A}_{i}^{r}\left(\dot{y}_{i}^{2}(t)+\frac{\mathscr{C}_{i}^{r}}{2 \mathscr{A}_{i}^{r}} y_{i}(t)\right)^{2}+\left(\mathscr{B}_{i}^{r}-\frac{\left(\mathscr{C}_{i}^{r}\right)^{2}}{4\left(\mathscr{A}_{i}^{r}\right)^{2}}\right) y_{i}^{2}(t)\right\}=0, \quad \forall t \in[0, \infty),
\end{aligned}
$$

that is,

$$
\dot{V}(t) \leq 0 .
$$

This implies that $V(t) \leq V(0), \forall t \in[0,+\infty)$ and

$$
\frac{1}{2} e^{2 r t} \sum_{i=1}^{n}\left\{b_{i} y_{i}^{2}(t)+\left(a_{i} \dot{y}_{i}(t)+c_{i} y_{i}(t)\right)^{2}\right\} \leq V(0) \text {. }
$$

Note that,

$e^{2 r t}\left(a_{i} \dot{y}_{i}(t)+c_{i} y_{i}(t)\right)^{2}=\left(a_{i} e^{r t} \dot{y}_{i}(t)+c_{i} e^{r t} y_{i}(t)\right)^{2}$.
That is

$$
a_{i}\left|\dot{y}_{i}(t)\right| e^{r t}=\left|a_{i} e^{r t} \dot{y}_{i}(t)+c_{i} e^{r t} y_{i}(t)\right|-\left|c_{i} y_{i}(t)\right| .
$$

Then, there exist an constant $M_{\phi, \psi}>0$ such that,

$$
\begin{aligned}
& \left|\dot{y}_{i}(t)\right| \leq M_{\phi, \psi} e^{-r t}, \\
& \left|y_{i}(t)\right| \leq M_{\phi, \psi} e^{-r t}, \quad \forall t \in[0,+\infty) .
\end{aligned}
$$

Hence from Definition 1 the system (9) is exponentially stable. 


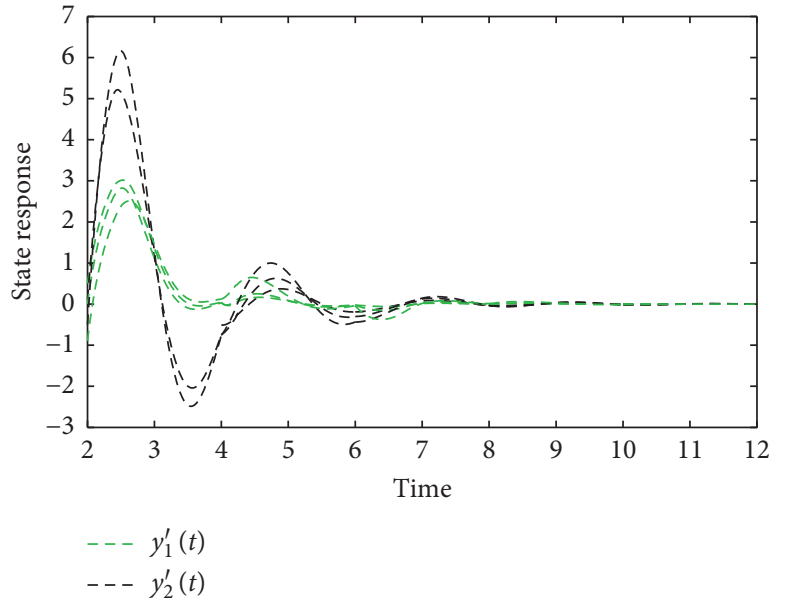

(a)

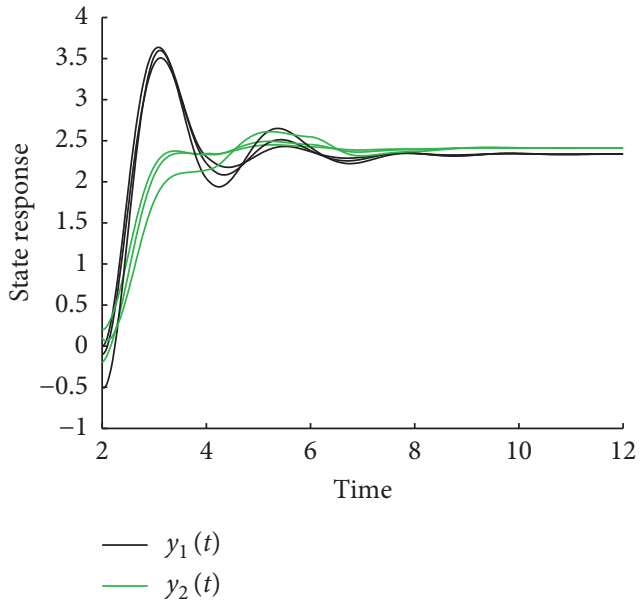

(b)

FIgURE 1: Exponential stability of (50) with $I_{i}=21$.

Corollary 2. Assume that the system (25) is exponentially stable if the assumption $\mathscr{R}_{1}$ and following conditions holds,

$$
\begin{aligned}
N_{i j}= & F_{j}\left(a_{i}^{2} \gamma_{i j}+a_{i} c_{i} \gamma_{i j}\right)+\eta_{i}\left(a_{i}^{2} \delta_{i j}+\delta_{i j}\left|a_{i} c_{i}\right|\right) e^{\left(1-\eta_{i}\right)} G_{j}+\eta_{i}\left(a_{i}^{2} \zeta_{i j}+\left|a_{i} c_{i}\right| \zeta_{i j}\right) H_{j} e^{2 r \tau_{i}} \tau_{i} \\
0> & a_{i} c_{i}-a_{i}^{2} \alpha_{i}+\frac{1}{2} \sum_{j=1}^{n} a_{i}^{2}\left[F_{j} \gamma_{i j}+G_{j} \delta_{i j}+H_{j} \zeta_{i j}\right] \\
0> & \left.-a_{i} c_{i} \beta_{i}+\frac{1}{2} \sum_{j=1}^{n} a_{j}^{2}\left[F_{j} \gamma_{i j}+G_{j} \delta_{i j}+H_{j} \zeta_{i j}\right]+\frac{1}{2}+G_{i} \delta_{j i}+H_{j} \zeta_{j i}\right]\left|a_{j} c_{j}\right|+\frac{1}{2} \sum_{j=1}^{n}\left[F_{j}\right. \\
& \times \sum_{j=1}^{n}\left[F_{i} \gamma_{j i} \gamma_{i j}+G_{j} \delta_{i j}+H_{j} \zeta_{i j}\right]\left|a_{i} c_{i}\right|
\end{aligned}
$$

Where $a_{i}, b_{i}$ and $c_{i}$ are positive constants, for all $i \in \mathbb{M}$.

Remark 3. In the stability theory, a Lyapunov functional plays a vital role. Basically, there are two approaches to fulfill the criteria of attaining the system stability with the Lyapunov functional (i.e. classical and traditional). In classical approach the construction of Lyapunov functional concerns only with the state variable of the proposed system, whereas in the case of traditional approach a Lyapunov functional that is not only contains the state variables but also the involvement of the derivatives of those state variables helps in attain the system stability much earlier. Moreover, the mathematical framework of the inertial neural networks involves with the second order differential equations which builts the computational complexity while manipulating, hence in order to avoid this burden, we utilized a traditional Lyapunov functional which is the great variation compared with the classical one.

\section{Numerical Simulation}

Example 1. Let us consider the following inertial neural networks with proportional delay, distributed delay and uncertain parameters as:

$$
\begin{aligned}
& \ddot{y}(t)=-(\alpha+\Delta \alpha(t)) \dot{y}(t)-(\beta+\Delta \beta) y(t)+(\gamma+\Delta \gamma) f(y(t))+(\delta+\Delta \delta) g(y(p t)) \\
& +(\zeta+\Delta \zeta) \int_{t-\sigma(t)}^{t} h(y(s)) \mathrm{d} s+I_{i} .
\end{aligned}
$$




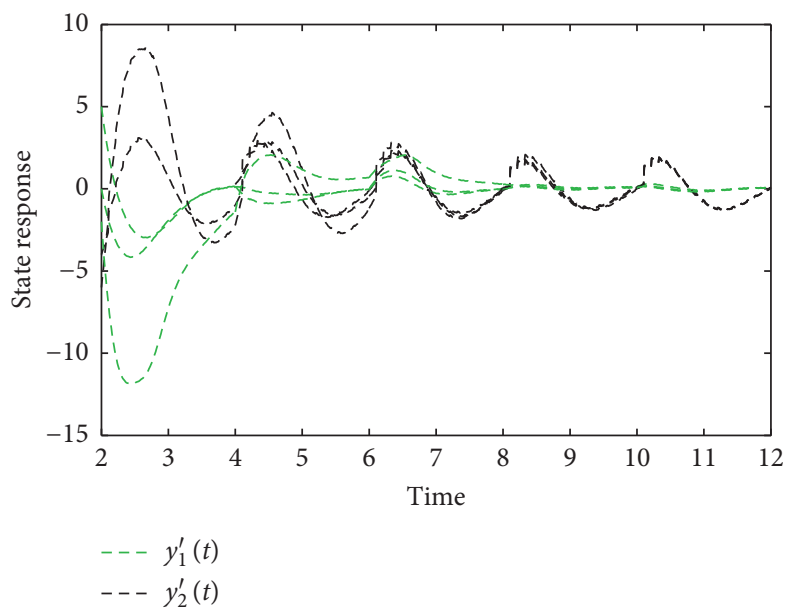

(a)

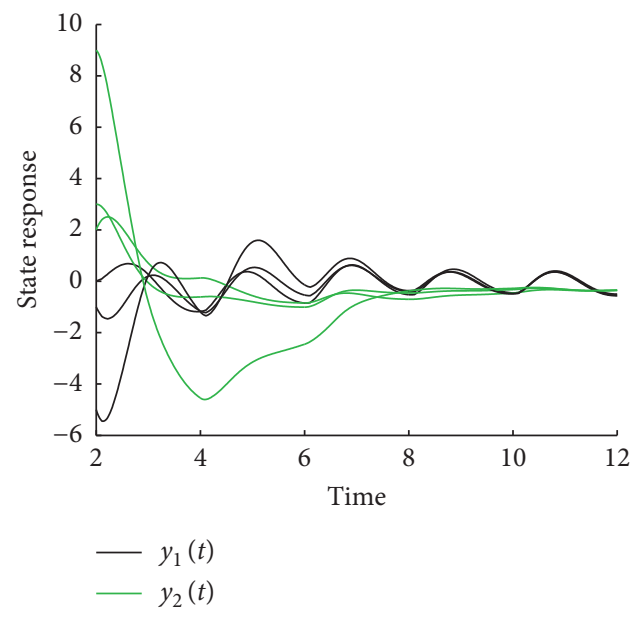

(b)

Figure 2: Exponential stability of (50) with $I_{i}=\sin t$.

Where

$$
\begin{aligned}
& y(t)=\left(y_{1}(t), y_{2}(t)\right)^{T} \\
& f_{j}=g_{j}=h_{j}=1.5|x-1| \text {; } \\
& \alpha=\left(\begin{array}{cc}
2 & 0 \\
0 & 1.75
\end{array}\right) \text {; } \\
& \beta=\left(\begin{array}{cc}
8 & 0 \\
0 & 2.83
\end{array}\right) \text {; } \\
& \gamma=\left(\begin{array}{cc}
-1 & 1.01 \\
-3.893 & 2.89
\end{array}\right) \text {; } \\
& \delta=\left(\begin{array}{cc}
-2.81 & -7.21 \\
-2.52 & 1.81
\end{array}\right) \text {; } \\
& \zeta=\left(\begin{array}{cc}
-3 & 0.81 \\
0.07 & -0.02
\end{array}\right) \text {; } \\
& \Delta \alpha(t)=\left(\begin{array}{cc}
-2.81 & -7.21 \\
-2.52 & 1.81
\end{array}\right) \text {; } \\
& \Delta \beta(t)=\left(\begin{array}{cc}
-2.81 & -7.21 \\
-2.52 & 1.81
\end{array}\right) \text {; } \\
& \Delta \gamma(t)=\left(\begin{array}{cc}
-2.81 & -7.21 \\
-2.52 & 1.81
\end{array}\right) \text {; } \\
& \Delta \delta(t)=\left(\begin{array}{cc}
-2.81 & -7.21 \\
-2.52 & 1.81
\end{array}\right) \\
& \Delta \zeta(t)=\left(\begin{array}{cc}
-2.81 & -7.21 \\
-2.52 & 1.81
\end{array}\right) \text {; } \\
& F_{j}=G_{j}=H_{j}=1.5 ; r=0.2 ; a_{1}=0.75, a_{2}=0.25 ; b_{1} \\
& =2.25, b_{2}=2.75 ; c_{1}=0.02, c_{2}=1.75 \text {; }
\end{aligned}
$$

we obtain

$$
\begin{aligned}
& A_{1}^{r}=-2.12 ; \\
& A_{2}^{r}=-29.4 ; \\
& B_{1}^{r}=-2.27 ; \\
& B_{2}^{r}=-31.22 ; \\
& C_{1}^{r}=-2.86 ; \\
& C_{2}^{r}=3.92 .
\end{aligned}
$$

Which implies that

$$
\begin{gathered}
A_{i}^{r}<0 \\
4 A_{i}^{r} B_{i}^{r}>\left(C_{i}^{r}\right)^{2}, \quad \text { for } i=1,2 .
\end{gathered}
$$

Clearly, all the conditions in Theorems 1 and 2 are satisfied, hence (50) is globally exponentially stable and has unique fixed point. Which was shown in Figures 1 and 2 with distinct initial condition and external inputs.

Remark 4. Figure 1 demonstrates the state trajectory of the system (9) in first and second order with constant external input and Figure 2 indicates the state trajectory of (9) with variable external input.

\section{Conclusion}

In this proposed work, we studied the existence, uniqueness and exponential stability of INNs with uncertain, proportional and distributed delays. Without shifting the order of the differential equation, under the Lyapunov functional method and a novel graph theoretic approach the necessary and the sufficient condition for stability of INNs was obtained. And by using the homeomorphism theorem the existence of an unique equilibrium point was derived. In the end of this work, a numerical simulations was presented to show the exactness of this proposed work. 
In future, we will discuss the synchronization of inertial neural networks and its applications to real life applications which were mentioned in the introductory section of our manuscript.

\section{Data Availability}

No data were used to support this study.

\section{Conflicts of Interest}

The authors declare that they have no conflicts of interest.

\section{Acknowledgments}

The article has been written with the joint partial financial support of the RUSA-Phase 2.0 grant sanctioned vide letter no. F.24-51/2014-U, policy (TN Multi-Gen), Dept. of Edn. Govt. of India, UGC-SAP (DRS-I) vide letter no. F.510/8/ DRS-I/2016(SAP-I), and DST (FIST- Phase I) vide letter no. SR/FIST/MS-I/2018-17 and the National Science Centre in Poland under Grant no. DEC-2017/25/B/ST7/02888 and J. Alzabut would like to thank Prince Sultan University for supporting this work.

\section{References}

[1] J. Dong, Z. Xia, W. Yan, and Q. Zhao, "Dynamic gesture recognition by directional pulse coupled neural networks for human-robot interaction in real time," Journal of Visual Communication and Image Representation, vol. 63, Article ID $102583,2019$.

[2] C. Vimala and P. A. Priya, "Artificial neural network based wavelet transform technique for image quality enhancement," Computers \& Electrical Engineering, vol. 76, pp. 258-267, 2019.

[3] W. Wang, X. Jia, X. Luo, J. Kurths, and M. Yuan, "Fixed-time synchronization control of memristive MAM neural networks with mixed delays and application in chaotic secure communication," Chaos, Solitons \& Fractals, vol. 126, pp. 85-96, 2019.

[4] L. Wang, X. Yu, T. Bourlai, and D. N. Metaxas, "A coupled encoder-decoder network for joint face detection and landmark localization," Image and Vision Computing, vol. 87, pp. 37-46, 2019.

[5] G. Zhang, J. Wang, C. Yan, and S. Wang, "Application research of image compression and wireless network traffic video streaming," Journal of Visual Communication and Image Representation, vol. 59, pp. 168-175, 2019.

[6] S. Zhu, L. Wang, and S. Duan, "Memristive pulse coupled neural network with applications in medical image processing," Neurocomputing, vol. 227, pp. 149-157, 2017.

[7] M. A. Cohen and S. Grossberg, "Absolute stability of global pattern formation and parallel memory storage by competitive neural networks," IEEE Transactions on Systems, Man, and Cybernetics, vol. 13, no. 5, pp. 815-826, 1983.

[8] J. Cao and J. Wang, "Global exponential stability and periodicity of recurrent neural networks with time delays," IEEE Transactions on Circuits and Systems I: Regular Papers, vol. 52, pp. 920-931, 2005.

[9] Y. Liu, P. Xu, J. Lu, and J. Liang, "Global stability of Cliffordvalued recurrent neural networks with time delays," Nonlinear Dynamics, vol. 84, no. 2, pp. 767-777, 2016.
[10] Z.-H. Guan and G. Chen, "On delayed impulsive Hopfield neural networks," Neural Networks, vol. 12, no. 2, pp. 273-280, 1999.

[11] J. Qi, C. Li, and T. Huang, "Stability of inertial BAM neural network with time-varying delay via impulsive control," Neurocomputing, vol. 161, pp. 162-167, 2015.

[12] X. Li and M. Bohner, "Exponential synchronization of chaotic neural networks with mixed delays and impulsive effects via output coupling with delay feedback," Mathematical and Computer Modelling, vol. 52, no. 5-6, pp. 643-653, 2010.

[13] C. M. Marcus and R. M. Westervelt, "Stability of analog neural networks with delay," Physical Review A, vol. 39, no. 1, pp. 347-359, 1989.

[14] J. Cao, D.-S. Huang, and Y. Qu, "Global robust stability of delayed recurrent neural networks," Chaos, Solitons \& Fractals, vol. 23, no. 1, pp. 221-229, 2005.

[15] C. Aouiti, "Neutral impulsive shunting inhibitory cellular neural networks with time-varying coefficients and leakage delays," Cognitive Neurodynamics, vol. 10, no. 6, pp. 573-591, 2016.

[16] R. Yang, B. Wu, and Y. Liu, "A Halanay-type inequality approach to the stability analysis of discrete-time neural networks with delays," Applied Mathematics and Computation, vol. 265, pp. 696-707, 2015.

[17] S. Aadhithiyan, R. Raja, Q. Zhu, J. Alzabut, M. Niezabitowski, and C. P. Lim, "Exponential synchronization of nonlinear multi-weighted complex dynamic networks with hybrid time varying delays," Neural Processing Letters, vol. 53, no. 2, pp. 1035-1063, 2021.

[18] M. S. Ali, M. Usha, Q. Zhu, and S. Shanmugam, "Synchronization analysis for stochastic T-S fuzzy complex networks with Markovian jumping parameters and mixed time-varying delays via impulsive control," Mathematical Problems in Engineering, vol. 2020, Article ID 9739876, 27 pages, 2020.

[19] K. Balasundaram, R. Raja, A. Pratap, and S. Chandrasekaran, "Impulsive effects on competitive neural networks with mixed delays: existence and exponential stability analysis," Mathematics and Computers in Simulation, vol. 155, pp. 290-302, 2019.

[20] Y. Xue, K. Chen, and K. Nahrstedt, “Achieving proportional delay differentiation in wireless LAN via crosslayer scheduling," Wireless Communications and Mobile Computing, vol. 4, no. 8, pp. 849-866, 2008.

[21] W. Pan, D. Mu, H. Wu, and Q. Sun, "Proportional delay differentiation service in web application aervers: a feedback control approach. In computing, communication, control, and management," CCCM 08 ISECS International Colloquium, vol. 1, pp. 600-604, 2008.

[22] R. Abazari and A. Kilıcman, "Application of differential transform method on nonlinear integro-differential equations with proportional delay," Neural Computing and Applications, vol. 24, no. 2, pp. 391-397, 2014.

[23] S. Jia, C. Hu, J. Yu, and H. Jiang, "Asymptotical and adaptive synchronization of Cohen-Grossberg neural networks with heterogeneous proportional delays," Neurocomputing, vol. 275, pp. 1449-1455, 2018.

[24] P. Wan, D. Sun, D. Chen, M. Zhao, and L. Zheng, "Exponential synchronization of inertial reaction-diffusion coupled neural networks with proportional delay via periodically intermittent control," Neurocomputing, vol. 01, pp. 1-16, 2019.

[25] L. Zhou, "Delay-dependent exponential stability of cellular neural networks with multi-proportional delays," Neural Processing Letters, vol. 38, no. 3, pp. 347-359, 2013. 
[26] R. Rao, Q. Zhu, and K. Shi, "Input-to-state stability for impulsive Gilpin-Ayala competition model with reaction diffusion and delayed feedback," IEEE Access, vol. 8, pp. 222625-222634, 2020.

[27] H. Shao and L. Shao, "A new Lyapunov-like functional approach to dwell-time dependent stability for impulsive systems," Journal of the Franklin Institute, vol. 357, no. 1, pp. 343-358, 2020.

[28] K. L. Babcock and R. M. Westervelt, "Stability and dynamics of simple electronic neural networks with added inertia," Physica D: Nonlinear Phenomena, vol. 23, no. 1-3, pp. 464469, 1986.

[29] K. L. Babcock and R. M. Westervelt, "Dynamics of simple electronic neural networks," Physica D: Nonlinear Phenomena, vol. 28, no. 3, pp. 305-316, 1987.

[30] D. W. Wheeler and W. C. Schieve, "Stability and chaos in an inertial two-neuron system," Physica D: Nonlinear Phenomena, vol. 105, no. 4, pp. 267-284, 1997.

[31] J. Wang and L. Tian, "Global Lagrange stability for inertial neural networks with mixed time-varying delays," Neurocomputing, vol. 235, pp. 140-146, 2017.

[32] P. Wan and J. Jian, "Global convergence analysis of impulsive inertial neural networks with time-varying delays," Neurocomputing, vol. 245, pp. 68-76, 2017.

[33] Q. Huang and J. Cao, "Stability analysis of inertial CohenGrossberg neural networks with Markovian jumping parameters," Neurocomputing, vol. 282, pp. 89-97, 2018.

[34] C. Maharajan, R. Raja, J. Cao, and G. Rajchakit, "Novel global robust exponential stability criterion for uncertain inertialtype BAM neural networks with discrete and distributed timevarying delays via Lagrange sense," Journal of the Franklin Institute, vol. 355, no. 11, pp. 4727-4754, 2018.

[35] Q. Tang and J. Jian, "Global exponential convergence for impulsive inertial complex-valued neural networks with timevarying delays," Mathematics and Computers in Simulation, vol. 159, pp. 39-56, 2019.

[36] F. Kong, Q. Zhu, and T. Huang, "New fixed-time stability lemmas and applications to the discontinuous fuzzy inertial neural networks," IEEE Transactions on Fuzzy Systems, vol. 1, 2020.

[37] Z. Chen, X. Wang, S. Zhong, and J. Yang, "Improved delaydependent robust passivity criteria for uncertain neural networks with discrete and distributed delays," Chaos, Solitons \& Fractals, vol. 103, pp. 23-32, 2017.

[38] N. Cui, H. Jiang, C. Hu, and A. Abdurahman, "Global asymptotic and robust stability of inertial neural networks with proportional delays," Neurocomputing, vol. 272, pp. 326-333, 2018.

[39] F. Kong, Q. Zhu, R. Sakthivel, and A. Mohammadzadeh, "Fixed-time synchronization analysis for discontinuous fuzzy inertial neural networks with parameter uncertainties," Neurocomputing, vol. 422, pp. 295-313, 2021.

[40] T. Saravanakumar, N. H. Muoi, and Q. Zhu, "Finite-time sampled-data control of switched stochastic model with nondeterministic actuator faults and saturation nonlinearity," Journal of the Franklin Institute, vol. 357, no. 18, pp. 13637$13665,2020$.

[41] F. Kong and Q. Zhu, "New fixed-time synchronization control of discontinuous inertial neural networks via indefinite Lyapunov-Krasovskii functional method," International Journal of Robust and Nonlinear Control, vol. 31, no. 2, pp. 471-495, 2020.

[42] Z.-M. Gao, Y. He, and M. Wu, "Improved stability criteria for the neural networks with time-varying delay via new augmented Lyapunov-Krasovskii functional," Applied Mathematics and Computation, vol. 349, pp. 258-269, 2019.

[43] M. Y. Li and Z. Shuai, "Global-stability problem for coupled systems of differential equations on networks," Journal of Differential Equations, vol. 248, no. 1, pp. 1-20, 2010.

[44] M. Forti and A. Tesi, "New conditions for global stability of neural networks with application to linear and quadratic programming problems," IEEE Transactions on Circuits and Systems I: Fundamental Theory and Applications, vol. 42, no. 7, pp. 354-366, 1995.

[45] X. He and W. Zhang, "Emotion recognition by assisted learning with convolutional neural networks," Neurocomputing, vol. 291, pp. 187-194, 2018. 